\title{
Experiment and Amount Optimization Analysis of Oil-Water Mixture Frying Machine Based on FLUENT
}

\author{
Xuejing Zhang ${ }^{1}$, Zhenwei $\mathrm{Yu}^{2}$, Khurram Yousaf ${ }^{2}$ and Kunjie Chen ${ }^{2 *}$ \\ ${ }^{1}$ Nanjing research Institute of Agricultural Mechanization, Ministry of Agriculture Nanjing 210014, China \\ ${ }^{2}$ College of Engineering, Nanjing Agricultural University Nanjing 210031, China \\ Corresponding author
}

\begin{abstract}
This paper simulated oil-heating process of frying machine by FLUENT software, in order to improve high-energy consumption problem of oil-water mixture frying machine in the working process. It analyzed temperature variation of nine key points, and came to a conclusion that temperature of different layers of frying machine has significant differences and temperature of each layer is even. Experimental results of nine key points are collected to be compared with the simulated results. According to comparison, the maximum temperature difference between experimental results of three points and simulated results is $5^{\circ} \mathrm{C}$; it at transition layer is $4^{\circ} \mathrm{C}$; it at oil-water interface is $3^{\circ} \mathrm{C}$. The results prove reliability of the model. Through comparing simulated results of different models, it can be concluded that when the distance from the interface to tank bottom is $120 \mathrm{~mm}$, the heating time is shortest; when the dimension of cooling tube is greater than or equal to $44 \mathrm{~mm}$, average temperature of frying layer stays in $180^{\circ} \mathrm{C}$; when power of a single heating tube is greater than $4 \mathrm{kw}$, although heating time still reduces slowly, heating consumption remains at $4.7 \times 103 \mathrm{kj}$.
\end{abstract}

Keywords-oil-water mixture; simulation; heat transfer; optimization design

\section{INTRODUCTION}

Oil-water mixture frying is a new technology, of which the essence is to add both water and oil in one frying tank when oil and water is naturally stratified because they have different specific gravity and they are incompatible. Oil is distributed at the upper layer of the frying tank because it has light specific gravity, and water at bottom due to heavy specific gravity[1]. At present, due to lack of researches on liquid heat transfer in frying tank of oil-water mixture frying machine, the knowledge to distribution of temperature of water and oil, and heat transfer in oil heating process is still blank, resulting in absence of reliable proof for optimization design of oil-water mixture frying machine and high energy consumption of the machine. Therefore, correct analysis on heat distribution and transfer of liquid in frying tank is of great significance to scientific manufacturing and optimization of oil-water mixture frying machine.

As for lack of researches on liquid heat transfer in frying tank, FLUENT software is applied to simulate oil heating process of frying machine, to analyze temperature distribution situation of oil and water in oil heating process, and to optimize or re-design all elements of frying machine by obtained results, so as to achieve the goal of energy conservation[2,3].

\section{Simulation Computation OF Oil HeAting PROCESS}

\section{A. Computational Domain and Meshing of Frying Machine}

The computational domain is created according to structural size of oil-water mixture frying machine, of which the structural diagram is as below. The frying machine's structure includes frying tank, shell body, filtering net, heating tube, cooling tube, oil outlet pipe, eject valve, and water intake pipe.

1. frying tank 2 . shell body 3 . filtering net 4 . heating tube 5 . oil outlet pipe 6 . eject valve 7 . water intake pipe 8 . Cool-water spraying pipe 9 . cooling tube

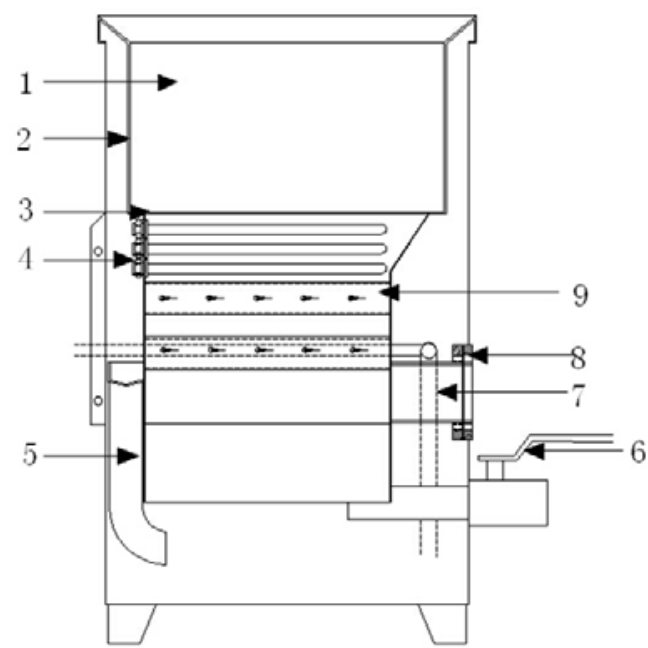

FIGURE I. STRUCTURAL DIAGRAM OF FRYING MACHINE

The geometric model built by ICEM CFD is as figure 2. In the model, height of middle-layer water is $140 \mathrm{~mm}$, and that of oil layer is $330 \mathrm{~mm} ; 3$ sets of heating tube in three rows are placed at $120 \mathrm{~mm}$ under oil surface, and its diameter is $10 \mathrm{~mm}$; power of each set of the heating tubes is $4 \mathrm{kw}$; diameter of cooling tube is $60 \mathrm{~mm}$, and there are 16 radiating tubes, which are placed in two rows. 


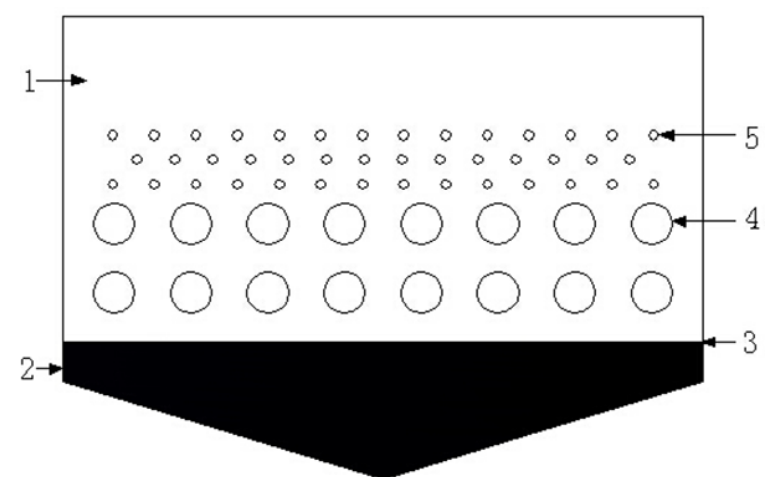

1. frying oil 2. water 3 .interface of water and oil 4 . Cooling tube 5 . Heating tube

FIGURE II. TWO-DIMENSIONAL STRUCTURAL MODEL OF FRYING MACHINE

Since the heating tubes in the model are quite close to each other, adaptable unstructured meshing method is applied[4]. Local refinement of heating tubes can help obtain higher precision while calculation speed is guaranteed[5].

\section{B. Simulation Parameters and Boundary Condition}

Here, energy equation, continuity equation, momentum equation (N-S) and k- $\varepsilon$ turbulence control equation are applied, and PISO is applied to solve the solution. Pressure option of spatial discretization is Standard, and the rest are default options. The initialization mode is Standard Initialization, to initialize flow field by whole computational domain, where convergence basis is residual error 10-5[6].

The essence of internal flow field of the frying machine is heat-driven closed-cycle flow field, and the method for place near the wall is near-wall equation. In the frying machine, there are two fluids, water and frying oil, which are tap-water and common soybean oil respectively. Heat-flow density of the heating tubes is $10465.43 \mathrm{kw} / \mathrm{m}^{3}[7]$. The surrounding walls of the frying tank is heat insulated. Initial temperature of oil and water is $26^{\circ} \mathrm{C}$.

\section{Internal Analysis on Computational Domain}

Transient simulation analysis by FLUENT is applied to simulate temperature distribution situation of oil and water during the heating and temperature elevating stage of the frying machine. Figure 3 is oil-water temperature distribution nephogram after 20-minute heating. From the figure, it is obvious that the whole temperature field is divided into three layers, among which the high-temperature layer is distributed at the frying layer, low-temperature layer at water layer, and the middle layer at transition layer. Main heat transfer model in the frying machine is thermal convection, which causes that temperature of frying layer is apparently higher than temperature of the other two layers. In actual frying process, frying layer should have enough high frying temperature, which should be about $180^{\circ} \mathrm{C}$. The simulated temperature of frying layer is in conformity with the actual oil temperature.

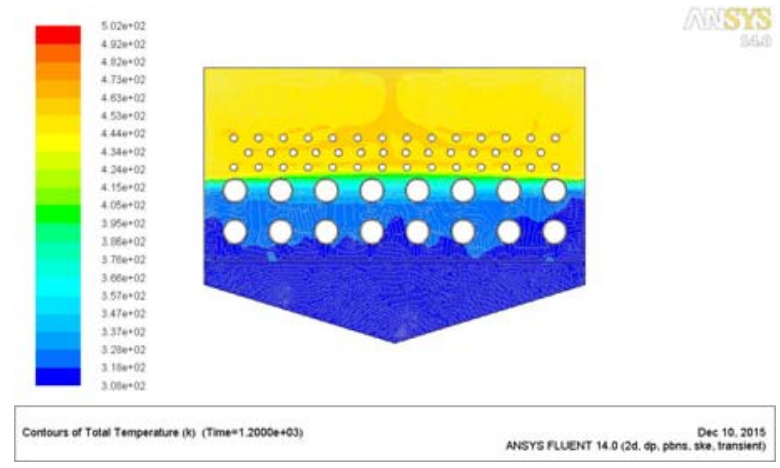

FIGURE III. TEMPERATURE DISTRIBUTION NEPHOGRAM OF FRYING MACHINE AFTER 20-MIN HEATING

\section{EXPERIMENT}

\section{A. Experiment Materials and Equipments}

The experiment materials include tap water and soybean oil, and experiment equipments are consisted of YZL-60 oil-water mixture frying machine, $\mathrm{K}$ thermocouple, voltage-stabilized source, 12-way temperature sample module, constant temperature digital bainmarie (for testing accuracy of thermocouple in sampling temperature), and PC (for real time display of temperature values and saved temperature data of nine key points).

\section{B. Experiment Plan}

The experiment aims at acquiring temperature values of key points, and using them for comparison with results of simulated computation. While in heating, oil-water mixture frying machine would have three layers of temperature, which are, high-temperature frying layer, oil-water transition layer, and low-temperature cooling layer. In order to compare temperature variation situations among three layers, the experiment personnel sets three key points at each layer (because this paper intends to influences of different positions at oil-water interface on temperature distribution of frying machine, the key points of low-temperature cooling layer is placed on the oil-water interface.). Please see figure 4.

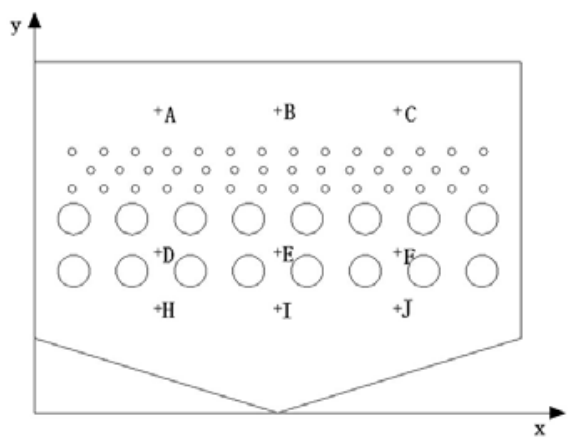

FIGURE IV. DISTRIBUTION OF KEY POINTS

\section{Verification on Numerical Model}

With heating power and structural size being constant, simulated computation results of nine points after $8 \mathrm{~min}$ ' $\mathrm{s}$ and 
20min's heating are read to be compared with experimental values, so as to verify reliability of the model. Comparison between simulated and experiment data is shown in figure 5 .

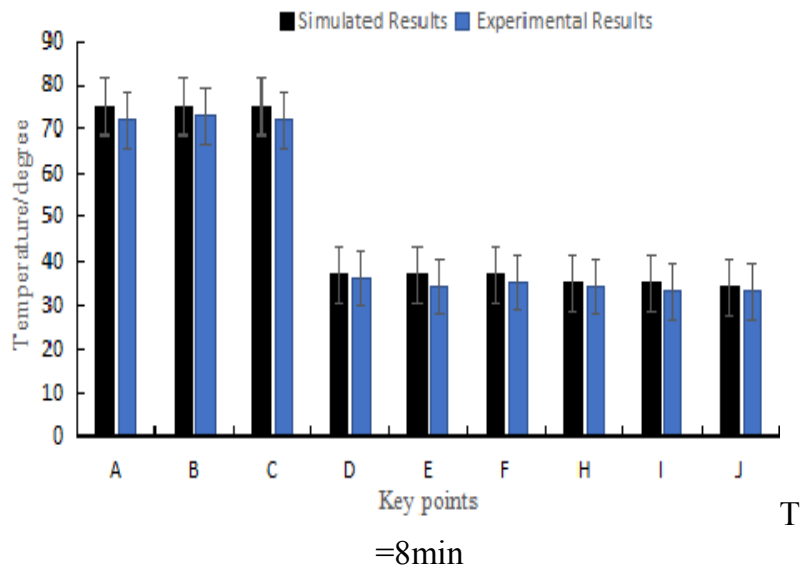

$\mathrm{T}(\mathrm{a})$

- Simulated Results $\square$ Experimental Results

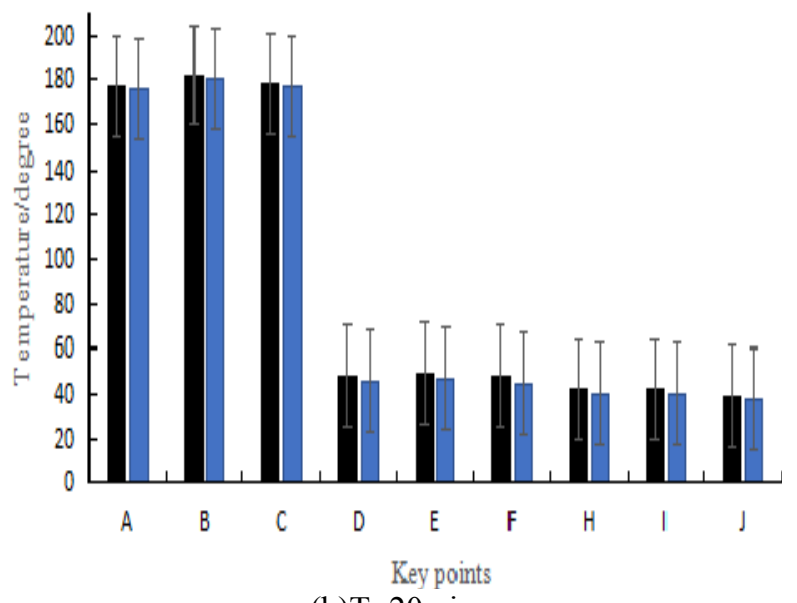

(b) $\mathrm{T}=20 \mathrm{~min}$

FIGURE V. TEMPERATURE COMPARISON OF KEY POINTS

Experimental values of temperature of nine points share same trend with simulated results, but experiment shows higher temperature values than simulated computation. The reason is that shell body of the frying machine is not completely heat insulated, but has heat leak in heating process. The maximum temperature difference between experimental results and simulated computation of three points at frying layer is $5^{\circ} \mathrm{C}$, and that at transition layer is $4^{\circ} \mathrm{C}$, and that at oil-water interface is $3^{\circ} \mathrm{C}$. The results prove that the selected numerical model and simulated plan are rational.

\section{RESULT ANALYSIS}

\section{A. Influence of Oil-water Mixture Proportion on Oil and} Water Temperature Distribution in Oil Heating Process

To study on influence of different oil-water mixture proportion on oil and water temperature distribution in oil heating process, heating processes of frying tank by 15 different oil-water mixture proportions are hereby simulated. Since the frying tank is not vertically symmetrical, oil-water mixture proportion cannot be shown directly by volume or quality ratio. Therefore, the distance (D) from oil-water interface to tank bottom is used to indicate different oil-water mixture proportion. The variable D is set to start from $100 \mathrm{~mm}$, with $10 \mathrm{~mm}$ interval, and to end at $240 \mathrm{~mm}$. Figure 6 is comparison of average temperature among frying layer, transition layer and interface under different positions of interface. From the temperature variation curve, it can be seen that temperature of transition layer goes higher as position of interface moves up. When D changes within 100 190mm, temperature variation at interface is relatively gentle. When $\mathrm{D}=120 \mathrm{~mm}$, the temperature of interface is the lowest, and it is $38^{\circ} \mathrm{C}$. When $\mathrm{D}>190 \mathrm{~mm}$, the temperature of interface gradually increases. When D changes within 100 190mm, temperature variation at frying layer is fluctuated. When $\mathrm{D}=120 \mathrm{~mm}$, the temperature of frying layer is highest and is $183.4^{\circ} \mathrm{C}$. When D $>190 \mathrm{~mm}$, the temperature of frying layer gradually increases.

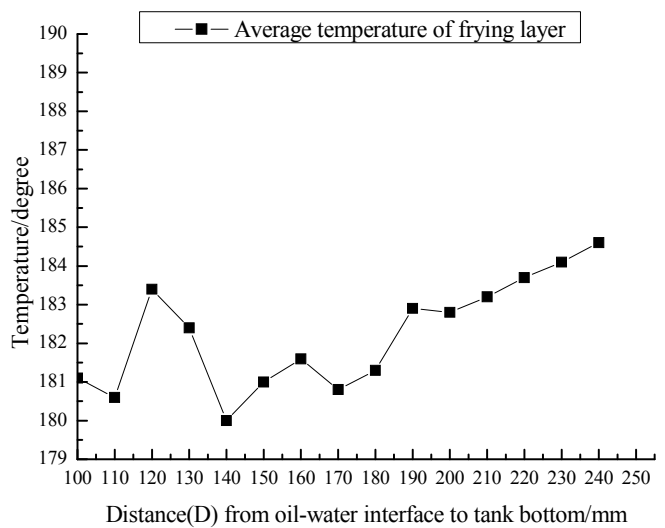

(a)

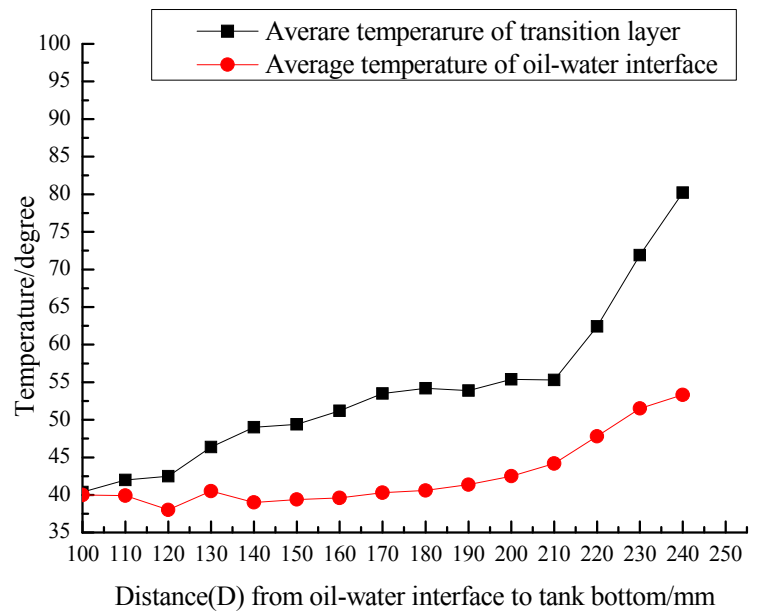

(b)

FIGURE VI. VARIATION CURVE OF AVERAGE TEMPERATURE IN DIFFERENT OIL-WATER MIXTURE PROPORTION 
When $\mathrm{D}=120 \mathrm{~mm}$, average temperature difference between frying layer and interface is largest, and the difference is $145.4{ }^{\circ} \mathrm{C}$. This mixture proportion not only ensures frying temperature of frying layer, but also reduces heating time, as well as decreases load of cooling device in frying stage. Therefore, when $\mathrm{D}=120 \mathrm{~mm}$, it is optimal position of oil-water interface.

\section{B. Influence of Different Diameters of Cooling Tube on Oil and Water Temperature Distribution in Oil Heating Process}

Cooling tube is a necessary component of oil-water mixture frying machine, and its function is to reduce the heat from heating layer to water layer and to ensure temperature of water is lower than safe temperature (to guarantee normal work of frying machine, temperature of water layer should be no more than $55^{\circ} \mathrm{C}$ ). To explore influence of difference diameters of cooling tube on oil and water temperature distribution, oil heating processes in 10 different diameters of cooling tube are hereby simulated. The 10 different diameters are $20 \mathrm{~mm}, 24 \mathrm{~mm}$, $28 \mathrm{~mm}, 32 \mathrm{~mm}, 36 \mathrm{~mm}, 40 \mathrm{~mm}, 44 \mathrm{~mm}, 48 \mathrm{~mm}, 52 \mathrm{~mm}, 56 \mathrm{~mm}$ and $60 \mathrm{~mm}$. Computational results of 10 models are respectively read, and average temperature values of frying layer and oil-water interface when each model is after 20mins' heating are acquired. The two layers' average temperature variation curves are shown in figure 7.

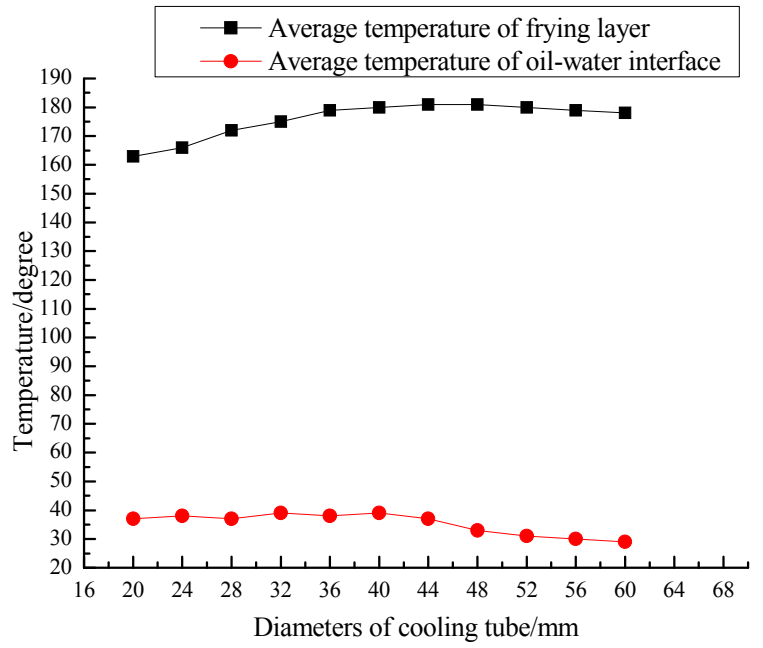

FIGURE VII. AVERAGE TEMPERATURE VARIATION CURVES OF FRYING LAYER AND INTERFACE IN DIFFERENT DIAMETERS OF COOLING TUBE

From the temperature variation curve, when diameter of the cooling tube is $\leq 44 \mathrm{~mm}$, average temperature of frying layer gradually increases, and that of interface remains at about $38^{\circ} \mathrm{C}$. When diameter of cooling tube is $\geq 44 \mathrm{~mm}$, variation range of average temperature of frying layer is small, and the average temperature stays in about $180^{\circ} \mathrm{C}$. In the meantime, average temperature of interface gradually decreases, to $29^{\circ} \mathrm{C}$ in minimum.
Bigger the diameter of cooling tube is, more space it would occupy. In addition, it would reduce adding amount of frying oil, and decrease heating time. However, if the diameter of cooling tube is too long, the distance between cooling tubes becomes small, causing greaves fall on the cooling tubes instead of the water layer. As for the frying machine discussed in this paper, the best diameter of cooling tube is $48 \mathrm{~mm}$.

\section{Influence of Different Power of Heating Tube on Oil and Water Temperature Distribution in Oil Heating Process}

Figure 8 shows variation of average temperature of transition layer and interface when average temperature of frying layer reaches to $180{ }^{\circ} \mathrm{C} \pm 1{ }^{\circ} \mathrm{C}$ under different heating power. The result indicates that heating power is $4.5 \mathrm{kw}$ when the average temperature of transition layer is lowest and it is $3.5 \mathrm{kw}$ when the average temperature of the interface is lowest. However, change of heating power would affect temperature distribution of all layers, and it also influences heating time and energy consumption. Figure 9 and Figure 10 respectively show required heating time and energy consumption when the frying layer is heated to $180^{\circ} \mathrm{C} \pm 1{ }^{\circ} \mathrm{C}$ under different heating power. From the curves, it can seen that heating time and consumed heat have sharp decline when heating power $(\mathrm{P})$ is increased from $1.5 \mathrm{kw}$ to $4.5 \mathrm{kw}$. When heating power is $>4.5 \mathrm{kw}$, although heating time still slowly reduces, consumed heat is in small variation and stays in about $4.7 \times 103 \mathrm{kj}$. In consideration of energy consumption saving, it is best when power of single heating tube is $4.5 \mathrm{kw}$.

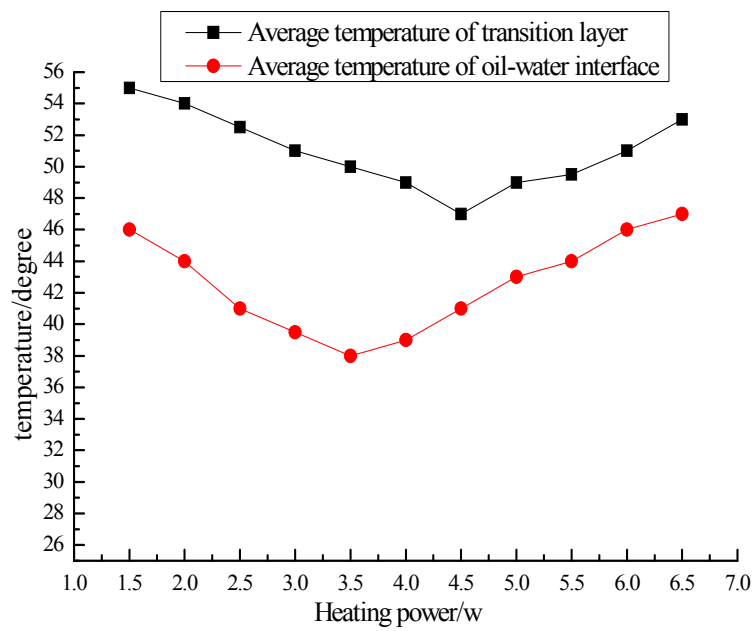

FIGURE VIII. VARIATION CURVE OF AVERAGE TEMPERATURE OF EACH LAYER UNDER $180^{\circ} \mathrm{C}$ AVERAGE TEMPERATURE OF FRYING LAYER 


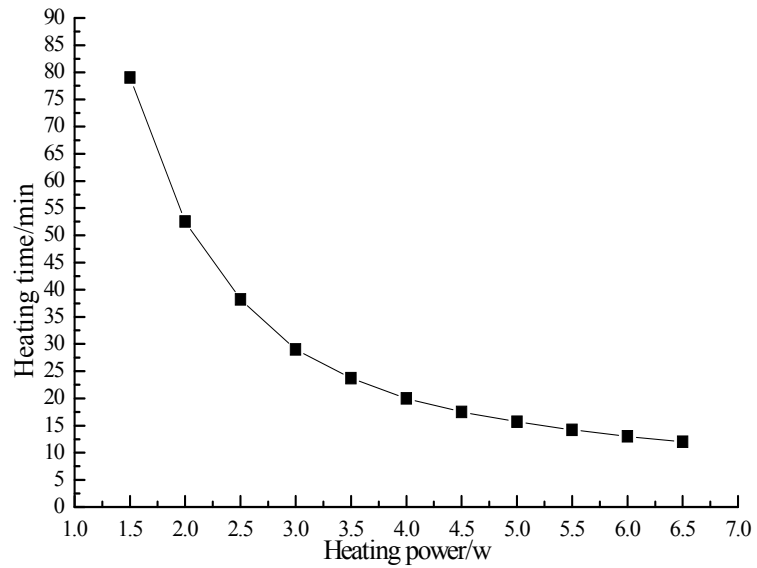

FIGURE IX. VARIATION CURVE OF HEATING TIME UNDER DIFFERENT HEATING POWER

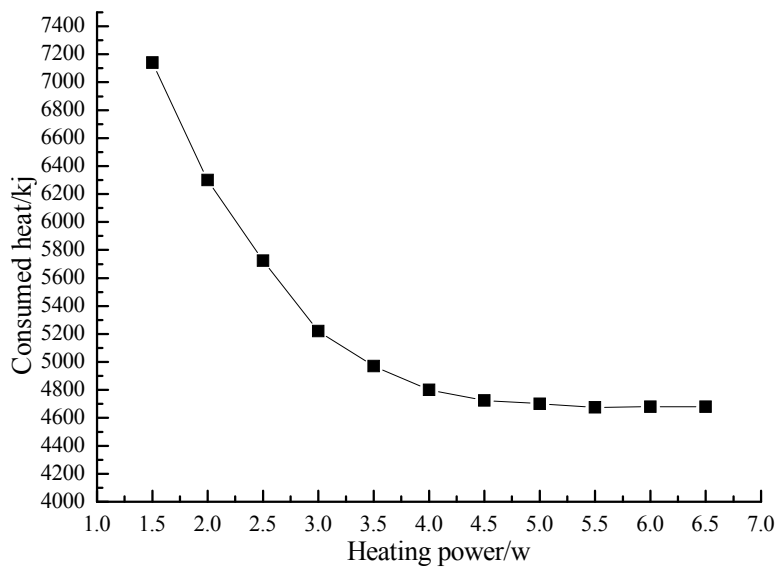

FIGURE X. VARIATION CURVE OF CONSUMED HEAT UNDER DIFFERENT HEATING POWER OF SINGLE HEATING TUBE

\section{CONCLUSION}

The maximum temperature difference between experiment test and simulated computation of three key points at frying layer is $5^{\circ} \mathrm{C}$, it at transition layer is $4^{\circ} \mathrm{C}$, and it at oil-water interface is $3{ }^{\circ} \mathrm{C}$. The results prove rationality of selected numerical model and simulation plan.

When the distance from interface to tank bottom is $120 \mathrm{~mm}$, temperature of frying layer increases fast, when that of transition layer and interface climbs slowly. This can shorten heating time and save energy consumption, and reduce load of cooling device in frying stage.

When diameter of cooling tube is $48 \mathrm{~mm}$, heat radiation is best, and greaves can go through cooling tube successfully.
When power of single heating tube is $4.5 \mathrm{kw}$, temperature of transition layer is lowest. it continues to ruse along with heating power, but it would reduce in heating time; consumed heat would have small variation in the period and remains in $4.7 \times 10^{3} \mathrm{kj}$.

\section{REFERENCES}

[1] An Jin.Optimal structure design of water-oil mixture frying machine[J].Food \& Machine.2008,06:97-98.

[2] Wang Weidong,Ping Sun,Zhang Rui,et al.Simulation of heat transfer by liquid-solid coupled method of engine block and cooling water jacket[J].Transaction of the CSAE,2010,26(7):118-122.

[3] Wang Fating,Lian Jinyi,Ning Hualong,et al.Research of Temperature Field in Asphalt Heating Tanks Based on FLUENT[J].Construction Machinery and Equipment.2013,11:30-34+4+3.

[4] Wu Lilong, Huang Meng, Chen Bing. A VOF Method Based on Unstructured Adaptive Grid[J].Journal of Engineering Thermophysics. 2011,11:1863-1865.

[5] XU.K,CHENG.Z.Q.Quality encoding for tetrahedral mesh optimization [J].Computer\& Graphics,2009,33(3):250-261.

[6] Zhou Liangfu,Fu Ximin,Xue Xinyu,et.al.Design and Experiment of Jet Mixing Apparatus Based on CFD $[\mathrm{J}]$.Transactions of the Chinese Society for Agricultural Machinery.2013,S1:107-112.

[7] Xiong Zhenqin,Wang Ruzhu,Wu Jingyi.Numerical Simulation of a Heat Pump Water Heater's Water Tank with Fluent Software[A].Recent advances in refrigeration and air conditioning-Fourth National Symposium on new technologies refrigeration and air conditioning [C].Southeast University,Shanghai Jiaotong University,2006:4. 